\title{
Guideline-Concordant Treatment Among Elderly Women With HER2-Positive Metastatic Breast Cancer in the United States
}

\author{
Ami M. Vyas, PhD, MS, MBA ${ }^{1}$; Hilary Aroke, MD, PhD, MPH${ }^{1}$; and Stephen Kogut, $\mathrm{PhD}, \mathrm{MBA}, \mathrm{RPh}^{1}$
}

\begin{abstract}
Background: It is crucial to identify whether women with HER2positive (HER2+) metastatic breast cancer (MBC) are treated according to treatment guidelines and whether treatment disparities exist. This study examined guideline-concordant treatment among women with HER2+ MBC and determined the magnitude of differences in treatment between those with positive and negative hormone receptor (HR) status using a nonlinear decomposition technique. Methods: A retrospective observational cohort study was conducted using the SEER-Medicare linked database. The study cohort consisted of women aged $\geq 66$ years diagnosed with HER2+ MBC in 2010 through $2013(n=241)$. Guideline-concordant initial treatment after cancer diagnosis was defined based on the NCCN Clinical Practice Guidelines in Oncology for Breast Cancer. A multivariable logistic regression was performed to identify significant predictors of guideline-concordant treatment. A postregression decomposition was conducted to identify the magnitude of disparities in treatment by HR status. Results: Of 241 women included in the study, a total of $76.8 \%$ received guideline-concordant treatment. These women were significantly more likely to have positive HR status $(P=.0298)$, have good performance status $(P=.0009)$, and more oncology visits $(P<.0001)$. With 1 -year increments in age at cancer diagnosis, the likelihood of receiving guideline-concordant treatment reduced by $5 \%(P=.0356)$. The decomposition analysis revealed that $19.0 \%$ of the disparity in guideline-concordant treatment between women with positive and negative HR status was explained by differences in their characteristics. Enabling characteristics (marital status, income, and education) explained the highest $(22.8 \%)$ proportion of the disparity. Conclusions: Nearly one-quarter of the study cohort did not receive guideline-concordant treatment. Our findings suggest opportunities to improve cancer care for elderly women with negative HR status who are unpartnered or have lower socioeconomic status. The high unexplained portion of the disparity by HR status can be due to patient treatment preferences, propensity to seek care, and organizational and physician-level characteristics that were not included in the study.
\end{abstract}

J Natl Compr Canc Netw 2020;18(4):405-413 doi: $10.6004 /$ jnccn.2019.7373

${ }^{1}$ Department of Pharmacy Practice, College of Pharmacy, University of Rhode Island, Kingston, Rhode Island.

\section{Background}

Cancer health disparity has received national attention and is considered a priority for health services research. Identifying cancer health disparities is critical to understanding why some patient groups may be more likely to not receive guideline-concordant care and die prematurely from cancer than their counterparts who received guideline-concordant care. Additionally, the US Department of Health \& Human Services has revealed its action plans to eliminate health disparities related to insurance coverage, quality of care, workforce diversity, population health, and data collection, and has geared its efforts and substantial national funding to meet its goals. ${ }^{1}$

Survival rates for breast cancer (BC), the most common cancer in US women, have been improving, ${ }^{2}$ although these improvements are not observed equally in all age groups, ${ }^{2}$ racial/ethnic groups, ${ }^{2,3}$ US regions, ${ }^{2}$ or socioeconomic groups. ${ }^{3-6}$

Approximately $20 \%$ of patients with BC have HER2positive (HER2+) tumors characterized by a more aggressive phenotype, leading to shorter times to relapse and adverse disease prognosis, ${ }^{7}$ and significantly shortened disease-free and overall survivals compared with those without HER2+ tumors. ${ }^{8,9}$ Additionally, women with metastases have a 5 -year survival rate of only $26 \%$, with almost none cured. ${ }^{10}$ Despite advances in the management of HER2 + metastatic breast cancer (MBC), the response rate to first-line treatment is $50 \%$ to $80 \%$, and $20 \%$ to $40 \%$ for second-line treatment, with most patients succumbing to their disease. ${ }^{11-15}$

Although standardized guidelines for the treatment of HER2 + MBC exist, few real-world studies have identified treatment patterns and opportunities to improve care among these patients. ${ }^{16-21}$ Two studies have evaluated guideline-concordant care. Poorvu et $\mathrm{al}^{20}$ used SEER-Medicare data for women diagnosed in 2010 through

See JNCCN.org for supplemental online content. 
2011 and found that approximately $20 \%$ of patients with HER2+ disease received no systemic therapy in the first 6 months after diagnosis. However, the HER2+ tumors were not categorized into hormone receptor (HR) status, which is an important determinant of treatment and disease prognosis. Another study also used SEER-Medicare data for women diagnosed in 2007 through 2013 and reported that among 96 women with HER2+ cancer, 42 received guideline-concordant care. ${ }^{21}$ Moreover, performance status (PS), an essential indicator of treatment decision in patients with advanced cancer, ${ }^{22}$ was not controlled for in these studies. Yet another study reported disparities in trastuzumab utilization, with worse overall survival among disparate patients. ${ }^{19}$ However, with no HER2 status data available in SEER before 2010, women who received trastuzumab were assumed to be HER2+. Additionally, Rugo et $\mathrm{al}^{18}$ reported racial/ethnic disparities in treatment patterns among patients with HER2+ MBC; however, the data were obtained between December 2003 and February 2006, and hence do not reflect recent trends in treatment of HER2+ MBC.

Approximately $45 \%$ of new BC cases are diagnosed in women aged $\geq 65$ years, with poor survival rates. ${ }^{23}$ Even though elderly women bear a higher BC burden and have worse outcomes, ${ }^{23}$ they remain underrepresented in many clinical trials. ${ }^{24-27}$ As a result, considerable controversy persists in what constitutes a guideline-concordant treatment plan for elderly women. There is a growing need to determine whether elderly women with HER2+ MBC are treated according to guidelines and if disparities exist. The primary objective of this study was to examine guideline-concordant treatment provided to elderly patients with BC with metastatic HER2 + tumor subtype using the SEER-Medicare database. The post hoc objective was to quantify the extent to which independent variables explained disparities in guideline-concordant treatment among women by their HR status using a nonlinear decomposition technique.

\section{Methods}

\section{Study Design and Data Source}

A retrospective observational cohort study was conducted using the SEER-Medicare linked database. The SEER program, which covers $26 \%$ of the US population, obtains information on patients newly diagnosed with cancer from 18 population-based tumor registries that in turn collect information from hospitals, outpatient clinics, laboratories, private practitioners, hospices, autopsy reports, and death certificates. $^{28}$ The Medicare data provide information on claims from inpatient, outpatient, physician, home health, durable medical equipment, and hospice care for individuals aged $\geq 65$ years who are enrolled in Medicare. The SEER cancer cases were linked to Medicare claims files. ${ }^{29}$ Details of the SEER-Medicare dataset are described elsewhere. ${ }^{28}$ For this study, the Area Resource File was linked to the SEER-Medicare dataset using the state and county level information to obtain census track data on income, education, and number of hospitals offering oncology services. ${ }^{30}$

\section{Study Cohort}

The study cohort consisted of women aged $\geq 66$ years at diagnosis of the first pathologically confirmed MBC (SEER site recode 46; AJCC stages IV, IV not otherwise specified [NOS], IVA, IVB, IVC) ${ }^{31}$ with HER2+ status in January 2010 through December 2013. Women with negative or unknown HER2 status and those diagnosed via a death certificate or autopsy were excluded, as were women who were enrolled in an HMO or were not continuously enrolled in Medicare Parts A and B during the study period of 12 months before cancer diagnosis through at least 6 months after diagnosis, death, or end of enrollment (Figure 1).

\section{Measures}

\section{Guideline-Concordant Treatment}

SEER data provide clinical information on HER2 status since 2010, which, along with HR status, was used to determine tumor status. Guideline-concordant initial treatment received within 6 months after cancer diagnosis ${ }^{20}$ per tumor status was determined based on the NCCN Clinical Practice Guidelines in Oncology (NCCN Guidelines) for patients with HER2+ MBC. Because women diagnosed between 2010 and 2013 were included, all versions of the NCCN Guidelines for BC published during this time frame were used to identify all possible relevant initial treatment strategies for the study cohort. $^{32-37}$ A woman was considered to have received guideline-concordant treatment if a regimen she obtained within 6 months of her cancer diagnosis matched a treatment listed in the NCCN Guidelines (supplemental eTable 1, available with this article at JNCCN.org). Medicare claims were used to identify endocrine therapies (using J-codes and National Drug Codes [NDC]), infused or oral chemotherapies (using J-codes and NDC), and HER2targeted therapies (using J-codes and NDC). Because the dose and duration of systemic therapy are not captured within Medicare Part B claims, evidence of the use of any systemic therapy recommended in the NCCN Guidelines was considered guideline-concordant treatment.

The primary measure of interest-receipt of guidelineconcordant treatment-was categorized as guidelineconcordant treatment and non-guideline-concordant treatment.

\section{Explanatory Variables}

The Andersen behavioral model of healthcare services utilization was used to evaluate significant predictors of 


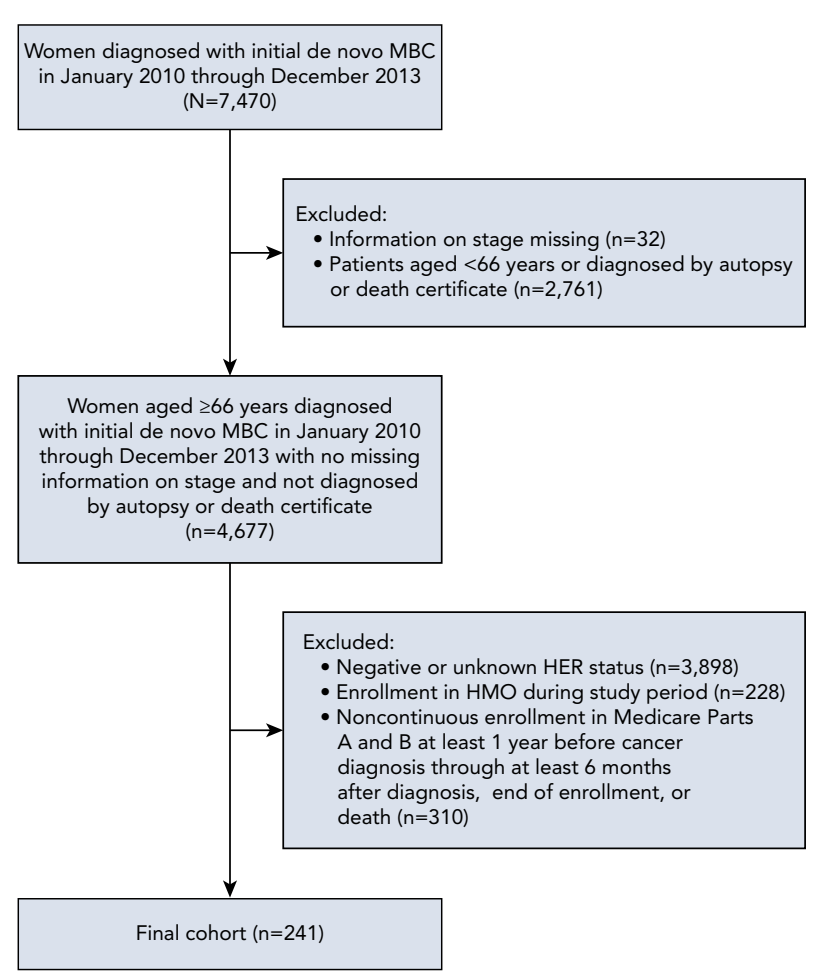

Figure 1. Patient flow diagram.

Abbreviations: $\mathrm{HMO}$, health maintenance organization; $\mathrm{MBC}$, metastatic breast cancer.

guideline-concordant treatment. ${ }^{38,39}$ This model has been widely used to understand healthcare services utilization in population-based studies. According to this model, healthcare utilization and medical care are functions of the predisposition of individuals to use services, factors that enable or impede use, the need for care, healthcare use, and external healthcare environmental factors. Predisposing factors included age at cancer diagnosis and race/ethnicity, and enabling factors included marital status, census tract median household income, and percentage of people age $\geq 25$ years with at least 4 years of college education, categorized as high or low based on the median value. Need-related factors included HR status, tumor grade, comorbidity scores ${ }^{40-42}$ derived from cooccurring chronic conditions within 12 months before diagnosis of BC, PS proxies, ${ }^{43}$ and number of sites with cancer metastasis. A proxy indicator for PS was developed by identifying claims associated with hospitalization, use of skilled nursing facility, oxygen use and related supplies, and use of wheelchair and walking aids in the year before cancer diagnosis. ${ }^{43}$ A woman with at least one claim for any of these services in the year before cancer diagnosis was considered to have poor PS, whereas a woman with no claim for any of these services was considered to have good PS. Healthcare use comprised medical oncology office visits during the follow-up period, ${ }^{44}$ and was categorized as low $(0-18)$ or high $(\geq 19)$ based on the median value. Medical oncology office visits were captured from the physician files through information describing provider specialty and date of office visit. Medical oncologists were defined as physicians with a listed specialty of medical oncology or hematology/oncology. The visits were determined using Healthcare Common Procedure Coding System codes for new office visits, established office visits, and office consultations listed in a previously published study. ${ }^{44} \mathrm{Ex}-$ ternal healthcare environmental factors included location of residence, SEER region, and census-level data on number of hospitals offering oncology services, categorized as low (0-2) or high ( $\geq 3$ ) based on the median value.

\section{Statistical Analyses}

Descriptive statistics were conducted to describe the study cohort. Chi-square tests were used to determine significant differences between the groups of women who received guideline-concordant treatment versus those who did not. A multivariable logistic regression with backward selection was performed to determine significant predictors of guidelineconcordant treatment among women with HER2+ MBC, after controlling for all the independent variables.

\section{Nonlinear Decomposition Technique}

BC molecular subtype is a key determinant in choosing appropriate cancer treatment. The NCCN Guidelines for $\mathrm{BC}$ have taken HR and HER status into consideration in guiding cancer care. ${ }^{33}$ In addition, from 2007 onward, ASCO recommended measuring HR status and HER2 expression for every primary invasive BC. ${ }^{45}$ Studies among patients with MBC have shown disparities in guideline-concordant care by HR status. ${ }^{20,21}$ Compared with patients with HER2+ MBC and HR-positive status, those with HR-negative disease have worse survival ${ }^{46}$ and hence typically require more aggressive treatment. In our study, the factors responsible for disparity in guidelineconcordant care by HR status needed to be identified and quantified. Therefore, to examine the magnitude of disparities in guideline-concordant treatment by HR status and the extent to which independent variables explained these disparities, a postregression nonlinear decomposition technique was used. ${ }^{47}$ In the report developed under contract from $\mathrm{NCI},{ }^{48}$ decomposition analysis is one of the methods identified to measure cancer disparities. With the nonlinear decomposition technique, the individual contribution of independent variables that explain the disparity across groups can be identified by calculating the change in the predicted probability obtained from replacing one independent variable at a time for one group and keeping all the other variables constant for the other group. The differences in guideline-concordant treatment by HR status were 
categorized into an explained portion and an unexplained portion. The explained portion provides disparities in guideline-concordant treatment by HR status that are caused by differences in the independent variables among the 2 groups. The unexplained portion provides disparities in treatment that could not be explained, either because of differences in the regression parameter estimates between the 2 groups or differences in unobservable and/or unmeasured determinants. Hence, the unexplained portion would remain even if the 2 groups had the same average levels of measured predictor variables. The explained portion of the gap is the sum of the differences between HR-positive and HR-negative women in terms of observed characteristics weighted by the pooled regression coefficients. It is calculated by multiplying the differences in the average characteristics between the 2 groups using pooled regression weights.

Analyses were conducted using SAS 9.4 (SAS Institute Inc). STATA, version 15.0 (StataCorp LLC) was used to perform nonlinear decomposition technique.

\section{Results}

\section{Descriptive Characteristics}

A total of 241 women aged $\geq 66$ years at first HER2 + MBC diagnosis in January 2010 through December 2013 were included in the study (see Figure 1). Most were aged $\geq 75$ years $(54.7 \%)$, white $(83.4 \%)$, and single, divorced, or widowed (74.3\%), and had HR-positive tumors (65.6\%), good PS (69.3\%), and at least 1 comorbidity $(63.9 \%)$ (Table 1$)$.

Within the study cohort, $76.8 \%$ received guidelineconcordant initial treatment and $23.2 \%$ did not. Women who received guideline-concordant treatment were significantly more likely to be aged 66 to 74 years, be married/partnered, reside in nonmetropolitan areas, have good PS, and have had more medical oncology visits. When treatment categories were examined among patients with HR-positive disease who received guidelineconcordant treatment, $36.2 \%$ received endocrine therapy only, $18.1 \%$ received endocrine therapy and HER2-targeted therapy, $22.1 \%$ received HER2-targeted therapy with or without chemotherapy, and $23.6 \%$ received all 3 therapies during the initial 6-month period after cancer diagnosis. Among patients with HR-negative MBC who received guideline-concordant treatment, $79.3 \%$ received HER2targeted therapy with chemotherapy, and $20.7 \%$ received HER2-targeted therapy only.

\section{Predictors of Guideline-Concordant Treatment}

After controlling for other predictive factors, women who were HR-positive were more than twice as likely to receive guideline-concordant treatment than those who were HR-negative (adjusted odds ratio [aOR], 2.234; 95\% CI, 1.082-4.615) (Table 2). With 1-year increments in age at cancer diagnosis, the likelihood of receiving guideline-concordant treatment reduced by $5 \%$ (aOR, 0.948; 95\% CI, 0.902-0.996). Compared with women who had poor PS, those with good PS were significantly more likely to receive guideline-concordant treatment (aOR, 3.345; 95\% CI, 1.634-6.849). Furthermore, women who had more medical oncology office visits were significantly more likely to receive guideline-concordant treatment (aOR, 8.076; 95\% CI, 3.509-18.591) than those with fewer medical oncology office visits.

\section{Factors Explaining Disparities in}

\section{Guideline-Concordant Treatment by HR Status}

There was a $10.5 \%$ difference in the proportion of women with HR-positive versus HR-negative status who received guideline-concordant treatment (Table 3). From the decomposition analysis, it was observed that of this difference, $2.0 \%$ was explained by the observed characteristics included in the study. Therefore, $19.0 \%$ of the disparity in guideline-concordant treatment between women with positive and negative HR status was explained by differences in these characteristics. Enabling factors explained the highest $(22.8 \%)$ proportion of this disparity, followed by external healthcare environmental factors at $5.3 \%$, needrelated factors at $3.2 \%$, and healthcare use at $-9.7 \%$. From the findings regarding enabling factors, it could be interpreted that if these factors were similar between women with positive and negative HR status, then the disparity in guideline-concordant treatment would decrease by $22.8 \%$. The negative coefficient of healthcare use indicates that if medical oncology office visits for women with positive and negative HR status were similar, then the disparity would increase by $9.7 \%$. Additionally, a large portion of the differences in guideline-concordant treatment by HR status remained unexplained (81.0\%).

\section{Discussion}

The seminal report Ensuring Quality of Cancer Care published in 1990 by the Institute of Medicine identified the need for research on cancer health disparities to optimize cancer care. ${ }^{49}$ Although this report created fervor, BC disparities still exist in the United States, which could be partly attributed to treatment disparities. Of several initiatives that have been designed to quantify and improve cancer care quality, ${ }^{50-52}$ a number of efforts have been targeted to non-MBC care. ${ }^{53-56}$ To the best of our knowledge, this study is the first of its kind to examine guideline-concordant treatment among elderly women with HER2+ MBC after controlling for a comprehensive list of confounders, including PS. In addition, we examined the extent to which predisposing, enabling, need-related, and external healthcare environmental factors and healthcare use explained disparities in guideline-concordant treatment by women's HR status. 


\section{Table 1. Patient Characteristics}

\begin{tabular}{|c|c|c|c|c|}
\hline Variables & $\begin{array}{c}\text { Total Cohort } \\
\text { n (\%) }\end{array}$ & $\begin{array}{c}\text { Guideline-Concordant } \\
\text { Treatment } \\
\text { n (\%) }\end{array}$ & $\begin{array}{c}\text { Non-Guideline-Concordant } \\
\text { Treatment } \\
\text { n (\%) }\end{array}$ & $P$ Value \\
\hline Total & $214(100)$ & $185(76.8)$ & $56(23.2)$ & \\
\hline Age at diagnosis, $y$ & & & & .0250 \\
\hline $66-74$ & $97(45.3)$ & $83(85.6)$ & $14(14.4)$ & \\
\hline $75-79$ & $56(18.2)$ & $41(73.2)$ & $15(26.8)$ & \\
\hline$\geq 80$ & $88(36.5)$ & $61(69.3)$ & $27(30.7)$ & \\
\hline Race/Ethnicity & & & & .1290 \\
\hline White & $201(83.4)$ & $158(78.6)$ & $43(21.4)$ & \\
\hline Other $^{\mathrm{a}}$ & $40(16.6)$ & $27(67.5)$ & $13(32.5)$ & \\
\hline Marital status & & & & .0100 \\
\hline Married/Partnered & $62(25.7)$ & $\mathrm{b}$ & $\mathrm{b}$ & \\
\hline Single/Divorced/Widowed & $179(74.3)$ & b & b & \\
\hline Location of residence & & & & .0500 \\
\hline Metropolitan & $189(78.4)$ & b & $\mathrm{b}$ & \\
\hline Nonmetropolitan & $52(21.6)$ & $\mathrm{b}$ & $\mathrm{b}$ & \\
\hline SEER region & & & & .8050 \\
\hline Northeast & $48(19.9)$ & $\mathrm{b}$ & b & \\
\hline South & $76(31.6)$ & $\mathrm{b}$ & b & \\
\hline North Central & $35(14.5)$ & $\mathrm{b}$ & b & \\
\hline West & $82(34.0)$ & $\mathrm{b}$ & $\mathrm{b}$ & \\
\hline Census tract household income (\$USD) & & & & .9990 \\
\hline$\leq \$ 50,000$ & $99(41.1)$ & $76(76.8)$ & $23(23.2)$ & \\
\hline$>\$ 50,000$ & $142(58.9)$ & $109(76.8)$ & $33(23.2)$ & \\
\hline Census tract education & & & & .6810 \\
\hline High $(>27.1)$ & $119(49.4)$ & $90(75.6)$ & $29(24.4)$ & \\
\hline Low (0-27.0) & $122(50.6)$ & $95(77.9)$ & $27(22.1)$ & \\
\hline Hormone receptor status & & & & .0670 \\
\hline Positive & $158(65.6)$ & $127(80.4)$ & $31(19.6)$ & \\
\hline Negative & $83(34.4)$ & $58(69.9)$ & $25(30.1)$ & \\
\hline Tumor grade & & & & .4080 \\
\hline Well/Moderately differentiated & $66(27.4)$ & $53(80.3)$ & $13(19.7)$ & \\
\hline Others & $175(72.6)$ & $132(75.4)$ & $43(24.6)$ & \\
\hline Number of metastatic sites ${ }^{c}$ & & & & .1320 \\
\hline $0-1$ & $170(70.5)$ & $126(74.1)$ & $44(25.9)$ & \\
\hline $2-4$ & $71(29.5)$ & $59(83.1)$ & $12(16.9)$ & \\
\hline Performance status & & & & .0004 \\
\hline Good & $167(69.3)$ & $139(83.2)$ & $28(16.8)$ & \\
\hline Poor & $74(30.7)$ & $46(62.2)$ & $28(37.8)$ & \\
\hline Comorbidity & & & & .1810 \\
\hline 0 & $87(36.1)$ & $71(81.6)$ & $16(18.4)$ & \\
\hline$\geq 1$ & $154(63.9)$ & $114(74.0)$ & $40(26.0)$ & \\
\hline Oncology visits & & & & $<.0001$ \\
\hline $0-18$ & $122(50.6)$ & $\mathrm{b}$ & b & \\
\hline$\geq 19$ & $119(49.4)$ & $\mathrm{b}$ & b & \\
\hline Hospitals offering oncology services & & & & .0250 \\
\hline $0-2$ & $122(50.6)$ & $101(82.8)$ & $21(17.2)$ & \\
\hline$\geq 3$ & $119(49.4)$ & $84(70.6)$ & $35(29.4)$ & \\
\hline
\end{tabular}

ancludes African American, American Indian/Alaskan Native, Asian or Pacific Islander, other unspecified, or unknown.

${ }^{b}$ Cells are suppressed because of small sample size that is in compliance with the SEER-Medicare data use agreement.

cMetastasis to brain, liver, lung, or bone. 


\begin{tabular}{|c|c|c|c|}
\hline Variable & aOR & $95 \% \mathrm{Cl}$ & $P$ Value \\
\hline Age at diagnosis & 0.948 & $(0.902-0.996)$ & .0356 \\
\hline \multicolumn{4}{|l|}{ Marital status } \\
\hline Married/Partnered & 2.193 & $(0.846-5.681)$ & .1060 \\
\hline Single/Divorced/Widowed & Ref & & \\
\hline \multicolumn{4}{|l|}{ HR status } \\
\hline Positive & 2.234 & $(1.082-4.615)$ & .0298 \\
\hline Negative & Ref & & \\
\hline \multicolumn{4}{|l|}{ Performance status } \\
\hline Good & 3.345 & $(1.634-6.849)$ & .0009 \\
\hline Poor & Ref & & \\
\hline \multicolumn{4}{|l|}{ Oncology visits } \\
\hline $0-18$ & Ref & & \\
\hline$\geq 19$ & 8.076 & (3.509-18.591) & $<.0001$ \\
\hline
\end{tabular}

Abbreviation: aOR, adjusted odds ratio.

${ }^{\text {aA }}$ multivariable logistic regression with backward selection was performed controlling for age at cancer diagnosis, race/ethnicity, marital status, location of residence, SEER region, census tract household income, census tract education, hormone receptor status, tumor grade, number of metastasis sites, performance status, comorbidity, oncology visits, and the number of hospitals offering oncology services. Findings from the fitted model are reported.

We found that $77 \%$ of elderly women with HER $2+$ MBC received guideline-concordant initial cancer treatment according to NCCN Guidelines, a marginally lower estimate than was reported earlier $(80 \%) .{ }^{20}$ However, our estimate was substantially higher than that reported in another study of HER2+ patients by Rocque et $\mathrm{al}^{21}$ ( $77 \%$ vs $44 \%$ ). However, such inconsistency could be because of the way HER2+ status was determined in that study - because no HER2 status data were available before 2010 , the authors categorized all women diagnosed before 2010 as an HER2-unknown group and considered them to be guideline-concordant if they received treatment for either HER2+ or HER2-negative MBC. A surprising finding was that nearly one-quarter of the study cohort did not receive initial cancer treatment according to recommended guidelines, thereby highlighting opportunities for improvement in the delivery of cancer care in older patients with cancer. The process of treatment decision-making in older patients with cancer is highly complex and multidimensional and comprises trade-offs resulting from increased risk of adverse events and functional decline after cancer treatment. ${ }^{57}$ In addition, an array of considerations can influence treatment decision-making, including patient-, physician-, and health system-related factors. A comprehensive geriatric assessment of older patients with cancer prior to oncologic decision-making can help clinicians detect previously unidentified health problems and provide the foundation for a treatment regimen specific to the needs of the patient..$^{58}$
Among predisposing factors, age at cancer diagnosis was significantly associated with receipt of guideline-concordant treatment. Increasing age was a significant predictor of nonreceipt of guidelineconcordant treatment in the multivariable analysis. This finding is similar to that reported earlier and may be attributed to several factors, including physician treatment choice and patient treatment preferences, which SEER-Medicare does not capture. ${ }^{20}$ Because cancer treatment may affect patient morbidity and quality of life, physicians may be conservative in choosing aggressive cancer treatment for relatively older patients compared with their younger counterparts. Furthermore, although elderly women are underrepresented in clinical trials, several subgroup analyses from recent trials have shown a good safety profile for recent targeted therapies, including pertuzumab and trastuzumab emtansine for elderly patients. ${ }^{59-61}$ Hence, age alone should not be a contraindication for cancer treatment.

Among need-related factors, negative HR status and poor PS were significant predictors of nonreceipt of guideline-concordant treatment. Our finding regarding negative HR status, consistent with that reported previously, ${ }^{21,62}$ indicates divergence from recommended care for women with negative HR status. Given that patients with HR-negative disease have poorer prognosis with shorter survival ${ }^{19}$ and benefit less from cancer treatment ${ }^{46}$ compared with those with positive HR status, physicians may be less likely to provide guideline-concordant treatment to these patients. Moreover, endocrine therapy, which has been the preferred first-line therapy for HRpositive disease, generally has fewer adverse effects and relatively more convenient routes of administration (eg, oral, intramuscular). Hence, patients with positive HR disease are more likely to receive guidelineconcordant care than those with negative HR disease.

Additionally, our finding about PS could be attributed either to physicians selecting less-aggressive treatments for elderly patients with poor PS or to preferences of patients seeking to avoid aggressive treatments. Our finding was similar to what was reported by a non-US study among patients with HER2+ MBC, ${ }^{63}$ thereby suggesting specific recommendations for this subgroup.

Nonlinear decomposition analysis showed that $19 \%$ of the disparity in guideline-concordant treatment between women with positive and negative HR status was explained by characteristics adjusted in the analyses. This method provides essential and crucial insights about guideline-concordant treatment in women with BC. Enabling factors, including marital status, household income, and education, accounted for the highest 
Table 3. Nonlinear Decomposition of Guideline-Concordant Treatment by HR Status

\begin{tabular}{|c|c|c|}
\hline & \multicolumn{2}{|c|}{ Guideline-Concordant Treatment } \\
\hline HR-negative disease & \multicolumn{2}{|c|}{$69.9 \%$} \\
\hline Total explained & \multicolumn{2}{|c|}{$2.0 \%$} \\
\hline Variable & $\begin{array}{l}\% \text { of Explained } \\
\text { Difference }\end{array}$ & $\begin{array}{l}\text { \% Contribution to the } \\
\text { Explained Portion }\end{array}$ \\
\hline $\begin{array}{l}\text { Predisposing factors (age at breast cancer } \\
\text { diagnosis, race/ethnicity) }\end{array}$ & $-2.6 \%$ & $-13.7 \%$ \\
\hline $\begin{array}{l}\text { Enabling factors (marital status, }{ }^{*} \text { census tract } \\
\text { median household income, }{ }^{\star \star} \text { and education) }\end{array}$ & $22.8 \%$ & $119.9 \%$ \\
\hline $\begin{array}{l}\text { Need-related factors (tumor grade, comorbidity, } \\
\text { performance status, }{ }^{\star \star} \text { number of sites of cancer } \\
\text { metastasis) }\end{array}$ & $3.2 \%$ & $17.0 \%$ \\
\hline Total difference explained & $19.0 \%$ & $100 \%$ \\
\hline Total difference unexplained & $81.0 \%$ & \\
\hline
\end{tabular}

Abbreviation: HR, hormone receptor.

a $P$ value of $<.20$ was used because of a relatively smaller sample size of HR-negative versus HR-positive patients.

${ }^{\star} P<.20$ a; ${ }^{* \star} P<.10$.

proportion of explained disparities in the recommended treatment between women with HR-positive and HRnegative cancers. A higher proportion of HR-negative patients were unmarried/single/divorced and had lower socioeconomic status (SES) compared with HRpositive patients (data not shown in tabular form), which explained $23 \%$ of the disparity in guidelineconcordant treatment. Our finding indicates that if women with negative HR status had the same marital and SES as those with positive HR status, then the disparity in guideline-concordant treatment would be reduced by almost $23 \%$. Our findings thereby suggest opportunities to address cancer care for women with negative HR status.

To our knowledge, no study has used a decomposition technique to study treatment disparities in patients with $\mathrm{BC}$, and hence it was difficult to compare our findings from decomposition analyses. However, our findings are interesting in the context of existing literature about social support and SES being associated with cancer treatment. Several studies have identified lack of social support ${ }^{20,64,65}$ and poorer SES $^{62,64,66,67}$ as the most common barriers to treatment of BC. However, none of these studies focused specifically on women with HER2+ metastatic disease. Cancer treatment often requires the availability of family members to facilitate care. Lack of family, friends, or living children may serve to limit access to cancer care. We did not anticipate identifying SES as a barrier to guideline-concordant treatment in women with negative HR status, because all women were covered by Medicare Parts A and B. However, the literature endorses that even insured patients with cancer can experience financial toxicity from substantial outof-pocket expenses, ${ }^{68}$ which may affect their receipt of recommended care.

A notable finding was that $81 \%$ of the difference in guideline-concordant treatment by HR status remained unexplained. It is likely that some portion of this difference could be attributed to severity of comorbid conditions, contraindications to cancer therapies, patient treatment preferences, propensity to seek care, physician treatment choice, and provider belief in the importance of guideline-concordance treatment, factors that are not captured in the SEER-Medicare database. The literature has also shown that several factors, including logistics of transportation and travel time to specialty physicians ${ }^{69}$-also not captured by the SEER-Medicare database-may cause disparities in access to healthcare.

A few limitations are worth noting. Some patientlevel variables, including annual household income, education level, and access to hospitals offering oncology services, are not available in the SEER-Medicare database, and therefore census tract information was used. ${ }^{70}$ Data on severity of comorbidities and patient symptoms, which may affect the selection of cancer treatment, were 
not captured. However, PS was determined using a claimsbased algorithm and was controlled for in the analyses. The study findings are generalizable only to elderly patients with cancer enrolled in Medicare. It is likely that many patients who did not receive guideline-concordant care received hospice care, and hospice use was not examined in our study. Moreover, a considerable number of women were excluded from the study because of their enrollment in an HMO or because of noncontinuous enrollment in Medicare Parts A and B during the study period, which may have affected our study findings and hence generalizability of our findings to all elderly women diagnosed with HER2+ MBC. Lastly, because of a smaller sample size of HR-negative patients, we used $P<.20$ for decomposition analysis. Future studies with larger sample size of HER2+ MBC patients are needed to validate our study findings.

\section{Conclusions}

Almost one-quarter of the study cohort did not receive guideline-concordant treatment. Our data from decomposition analysis suggest opportunities to address cancer care for elderly women with negative HR status.

Submitted April 5, 2019; accepted for publication October 23, 2019.

Previous presentation: Results of these analyses were presented at the NCCN 2019 Annual Conference; March 21-23, 2019; Orlando, Florida. Abstract HSR19-111.

Author contributions: Study concept and design: Vyas, Kogut. Data collection: Vyas. Data analysis: Vyas, Aroke. Data interpretation: All authors. Manuscript preparation: All authors. Review and approval of final manuscript: All authors.

Disclosures: The authors have disclosed that they have not received any financial consideration from any person or organization to support the preparation, analysis, results, or discussion of this article.

Funding: This study was supported by the pilot award received by Dr. Vyas via Institutional Development Award (IDeA) Network for Biomedical Research Excellence from the National Institute of General Medical Sciences (NIGMS) of the NIH (grant P20GM103430). Dr. Kogut is partially supported by Institutional Development award (U54GM115677) from the NIGMS of the NIH which funds Advance Clinical and Translational Research (Advance-CTR). The content is solely the responsibility of the authors and does not necessarily represent the official views of NIGMS.

Correspondence: Ami M. Vyas, PhD, MS, MBA, Department of Pharmacy Practice, College of Pharmacy, University of Rhode Island, 7 Greenhouse Road, Kingston, RI 02881. Email: avyas@uri.edu

\section{References}

1. Koh HK, Graham G, Glied SA. Reducing racial and ethnic disparities: the action plan from the Department of Health and Human Services. Health Aff (Millwood) 2011;30:1822-1829.

2. Howlader $N$, Noone AM, Krapcho $M$, et al, eds. SEER cancer statistics review, 1975-2014, National Cancer Institute. Bethesda, MD. Available at: http:// seer.cancer.gov/csr/1975 2014/, based on November 2016 SEER data submission, posted to the SEER web site, April 2017. Accessed March 12, 2019

3. Bradley CJ, Given CW, Roberts C. Race, socioeconomic status, and breast cancer treatment and survival. J Natl Cancer Inst 2002;94:490-496.

4. Yu XQ. Socioeconomic disparities in breast cancer survival: relation to stage at diagnosis, treatment and race. BMC Cancer 2009;9:364.

5. Ward E, Jemal A, Cokkinides V, et al. Cancer disparities by race/ethnicity and socioeconomic status. CA Cancer J Clin 2004;54:78-93.

6. Parise CA, Caggiano V. Disparities in race/ethnicity and socioeconomic status: risk of mortality of breast cancer patients in the California Cancer Registry, 2000-2010. BMC Cancer 2013;13:449.

7. Ross JS, Slodkowska EA, Symmans WF, et al. The HER-2 receptor and breast cancer: ten years of targeted anti-HER-2 therapy and personalized medicine. Oncologist 2009;14:320-368

8. Slamon DJ, Clark GM, Wong SG, et al. Human breast cancer: correlation of relapse and survival with amplification of the HER-2/neu oncogene. Science 1987;235:177-182.

9. Slamon DJ, Godolphin W, Jones LA, et al. Studies of the HER-2/neu protooncogene in human breast and ovarian cancer. Science 1989;244: 707-712.

10. Siegel RL, Miller KD, Jemal A. Cancer Statistics, 2017. CA Cancer J Clin 2017:67:7-30.

11. Slamon DJ, Leyland-Jones $B$, Shak $S$, et al. Use of chemotherapy plus a monoclonal antibody against HER2 for metastatic breast cancer that overexpresses HER2. N Engl J Med 2001;344:783-792.

12. Baselga J, Cortés J, Kim SB, et al. Pertuzumab plus trastuzumab plus docetaxel for metastatic breast cancer. N Engl J Med 2012;366: 109-119.

13. Verma S, Miles D, Gianni L, et al. Trastuzumab emtansine for HER2positive advanced breast cancer. N Engl J Med 2012;367:1783-1791.

14. Geyer CE, Forster J, Lindquist D, et al. Lapatinib plus capecitabine for HER2-positive advanced breast cancer. N Engl J Med 2006;355: 2733-2743.

15. Swain SM, Kim SB, Cortés J, et al. Pertuzumab, trastuzumab, and docetaxel for HER2-positive metastatic breast cancer (CLEOPATRA study): overall survival results from a randomised, double-blind, placebocontrolled, phase 3 study. Lancet Oncol 2013;14:461-471.

16. Lang $\mathrm{K}, \mathrm{Hao} Y$, Huang $\mathrm{H}$, et al. Treatment patterns among elderly patients with stage IV breast cancer treated with HER-2-targeted therapy. J Comp Eff Res 2014;3:481-490.

17. Taylor DC, Sanon M, Clements K, et al. Treatment patterns and costs following metastatic breast cancer diagnosis in U.S. women: a SEER-medicare analysis [abstract]. J Clin Oncol 2011;29(Suppl): Abstract 150

18. Rugo HS, Brufsky AM, Ulcickas Yood M, et al. Racial disparities in treatment patterns and clinical outcomes in patients with HER2positive metastatic breast cancer. Breast Cancer Res Treat 2013;141: 461-470.

19. Vaz-Luis I, Lin NU, Keating NL, et al. Racial differences in outcomes for patients with metastatic breast cancer by disease subtype. Breast Cancer Res Treat 2015;151:697-707.

20. Poorvu PD, Vaz-Luis I, Freedman RA, et al. Variation in guidelineconcordant care for elderly patients with metastatic breast cancer in the United States. Breast Cancer Res Treat 2018;168:727-737.

21. Rocque GB, Williams CP, Jackson BE, et al. Impact of nonconcordance with NCCN guidelines on resource utilization, costs, and mortality in de novo metastatic breast cancer. J Natl Compr Canc Netw 2018;16: 1084-1091.

22. Karnofsky DA, Burchenal JH. The clinical evaluation of chemotherapeutic agents in cancer. In: MacLeod CM, ed. Evaluation of Chemotherapeutic Agents. New York, NY: Columbia University Press; 1949:191-205.

23. American Cancer Society. Cancer facts \& figures 2019. Available at: https://www.cancer.org/content/dam/cancer-org/research/cancer-factsand-statistics/annual-cancer-facts-and-figures/2019/cancer-facts-andfigures-2019.pdf. Accessed March 12, 2019

24. Hutchins LF, Unger JM, Crowley JJ, et al. Underrepresentation of patients 65 years of age or older in cancer-treatment trials. N Engl J Med 1999;341: 2061-2067.

25. Kemeny $M M$, Peterson $B L$, Kornblith $A B$, et al. Barriers to clinical trial participation by older women with breast cancer. J Clin Oncol 2003;21: 2268-2275.

26. Hurria A, Dale W, Mooney M, et al. Designing therapeutic clinical trials for older and frail adults with cancer: U13 conference recommendations. J Clin Oncol 2014;32:2587-2594

27. Freedman RA. Treatment of breast cancer in the elderly. Curr Oncol Rep $2015 ; 17: 51$ 
28. Warren JL, Klabunde CN, Schrag D, et al. Overview of the SEER-Medicare data: content, research applications, and generalizability to the United States elderly population. Med Care 2002;40(8 Suppl):IV-3-18.

29. National Cancer Institute, Division of Cancer Control \& Population Sciences. SEER-Medicare: about the data files. Available at: https:// healthcaredelivery.cancer.gov/seermedicare/aboutdata/. Accessed March 12, 2019.

30. U.S. Health Resources and Services Administration, Bureau of Health Professions. Area Resource File, 2009-2010 Release. Rockville, MD: US Department of Health and Human Services; Fairfax, VA: Quality Resource Systems, Inc; 2010. Codebook: HE-001(2010). Available at: https://data. hrsa.gov/topics/health-workforce/ahrf. Accessed March 12, 2019.

31. Greene FL, Page DL, Fleming ID, et al., eds. AJCC Cancer Staging Manual. 6th ed. New York, NY: Springer; 2002.

32. Gradishar WJ, Anderson BO, Blair SL, et al. Breast cancer version 3.2014. J Natl Compr Canc Netw 2014;12:542-590.

33. Theriault RL, Carlson RW, Allred C, et al. Breast cancer, version 3.2013: featured updates to the NCCN guidelines. J Natl Compr Canc Netw 2013; 11:753-760 [quiz 761].

34. Carlson RW, Allred DC, Anderson BO, et al. Metastatic breast cancer, version 1.2012: featured updates to the NCCN guidelines. J Natl Compr Canc Netw 2012;10:821-829

35. Carlson RW, Allred DC, Anderson BO, et al. Breast cancer. Clinical practice guidelines in oncology. J Natl Compr Canc Netw 2009;7:122-192.

36. Carlson RW, Allred DC, Anderson BO, et al. NCCN Clinical Practice Guidelines in Oncology: Breast Cancer. Version 1.2010. Obtained with permission from NCCN January 1, 2018. To view the most recent version, visit NCCN.org.

37. Carlson RW, Allred C, Anderson BO, et al. NCCN Clinical Practice Guidelines in Oncology. Breast Cancer. Version 1.2011. Obtained with permission from NCCN January 1, 2018. To view the most recent version, visit NCCN.org.

38. Andersen R, Newman JF. Societal and individual determinants of medical care utilization in the United States. Milbank Mem Fund Q Health Soc 1973;51:95-124.

39. Andersen RM. Revisiting the behavioral model and access to medical care: does it matter? J Health Soc Behav 1995;36:1-10.

40. Klabunde CN, Potosky AL, Legler JM, et al. Development of a comorbidity index using physician claims data. J Clin Epidemiol 2000;53: 1258-1267.

41. National Cancer Institute. SEER-Medicare: Selection the appropriate comorbidity SAS Macro. Available at: https://healthcaredelivery.cancer.gov/ seermedicare/considerations/calculation.html. Accessed July 12, 2019.

42. Charlson ME, Pompei $P$, Ales $K L$, et al. A new method of classifying prognostic comorbidity in longitudinal studies: development and validation. J Chronic Dis 1987;40:373-383.

43. Onukwugha E, Qi R, Jayasekera J, et al. Cost prediction using a survival grouping algorithm: An application to incident prostate cancer cases. Pharmacoeconomics 2016;34:207-216.

44. Accordino MK, Wright JD, Vasan S, et al. Use and costs of disease monitoring in women with metastatic breast cancer. J Clin Oncol 2016;34: 2820-2826.

45. Harris L, Fritsche H, Mennel R, et al. American Society of Clinical Oncology 2007 update of recommendations for the use of tumor markers in breast cancer. J Clin Oncol 2007;25:5287-5312.

46. Lobbezoo DJ, van Kampen RJW, Voogd AC, et al. Prognosis of metastatic breast cancer subtypes: the hormone receptor/HER2-positive subtype is associated with the most favorable outcome. Breast Cancer Res Treat 2013;141:507-514.

47. Fairlie RW. An extension of the blinder-oaxaca decomposition technique to logit and probit models. J Econ Soc Meas 2005;30(4):305-316.

48. Harper S, Lynch J. Methods for measuring cancer disparities: using data relevant to Healthy People 2010 cancer-related objectives. Available at: https://seer.cancer.gov/archive/publications/disparities/measuring disparities.pdf. Accessed August 1, 2019

49. Hewitt M, Simone JV, eds. Ensuring the Quality of Cancer Care. Washington, DC: National Academy Press; 1999
50. Neuss MN, Desch CE, McNiff KK, et al. A process for measuring the quality of cancer care: the Quality Oncology Practice Initiative. J Clin Oncol 2005;23:6233-6239.

51. Hassett MJ, Schymura MJ, Chen K, et al. Variation in breast cancer care quality in New York and California based on race/ethnicity and Medicaid enrollment. Cancer 2016;122:420-431.

52. Malin JL, Schuster MA, Kahn KA, et al. Quality of breast cancer care: what do we know? J Clin Oncol 2002;20:4381-4393.

53. Malin JL, Asch SM, Kerr EA, et al. Evaluating the quality of cancer care: development of cancer quality indicators for a global quality assessment tool. Cancer 2000;88:701-707.

54. Earle CC, Burstein HJ, Winer EP, et al. Quality of non-breast cancer health maintenance among elderly breast cancer survivors. J Clin Oncol 2003;21: 1447-1451.

55. Malin JL, Schneider EC, Epstein AM, et al. Results of the National Initiative for Cancer Care Quality: how can we improve the quality of cancer care in the United States? J Clin Oncol 2006;24:626-634.

56. Hassett MJ, Hughes ME, Niland JC, et al. Selecting high priority quality measures for breast cancer quality improvement. Med Care 2008;46: 762-770.

57. Soto-Perez-de-Celis E, Li D, Yuan Y, et al. Functional versus chronological age: geriatric assessments to guide decision making in older patients with cancer. Lancet Oncol 2018;19:e305-316.

58. Festen S, Kok M, Hopstaken JS, et al. How to incorporate geriatric assessment in clinical decision-making for older patients with cancer. An implementation study [published April 26, 2019]. J Geriatr Oncol, doi: 10.1016/j.jgo.2019.04.006.

59. Miles D, Baselga J, Amadori D, et al. Treatment of older patients with HER2-positive metastatic breast cancer with pertuzumab, trastuzumab, and docetaxel: subgroup analyses from a randomized, double-blind placebo-controlled phase III trial (CLEOPATRA). Breast Cancer Res Treat 2013;142:89-99.

60. Le Saux O, Falandry C, Gan HK, et al. Inclusion of elderly patients in oncology clinical trials. Ann Oncol 2016;27:1799-1804.

61. Montemurro F, Ellis $\mathrm{P}$, Anton $\mathrm{A}$, et al. Safety of trastuzumab emtansine (T-DM1) in patients with HER2-positive advanced breast cancer: primary results from the KAMILLA study cohort 1. Eur J Cancer 2019; 109:92-102.

62. Akinyemiju T, Moore JX, Ojesina Al, et al. Racial disparities in individual breast cancer outcomes by hormone-receptor subtype, area-level socioeconomic status and healthcare resources. Breast Cancer Res Treat 2016 157:575-586

63. Colomer R, Hall P, Szkultecka-Debek M, et al. Real-world treatment in patients with HER2 + metastatic breast cancer: treatment decisions in HER2 + mBC. Breast Cancer Res Treat 2018;168:197-205.

64. Hendren S, Chin N, Fisher S, et al. Patients' barriers to receipt of cancer care, and factors associated with needing more assistance from a patient navigator. J Natl Med Assoc 2011;103:701-710.

65. Osborne C, Ostir GV, Du X, et al. The influence of marital status on the stage at diagnosis, treatment, and survival of older women with breast cancer. Breast Cancer Res Treat 2005;93:41-47.

66. Dreyer MS, Nattinger AB, McGinley EL, Pezzin LE. Socioeconomic status and breast cancer treatment. Breast Cancer Res Treat 2018; 167:1-8.

67. Wu XC, Lund MJ, Kimmick GG, et al. Influence of race, insurance, socioeconomic status, and hospital type on receipt of guideline-concordant adjuvant systemic therapy for locoregional breast cancers. J Clin Oncol 2012;30:142-150.

68. Davidoff AJ, Erten M, Shaffer T, et al. Out-of-pocket health care expenditure burden for Medicare beneficiaries with cancer. Cancer 2013; 119:1257-1265

69. Devoe JE, Baez A, Angier H, et al. Insurance + access not equal to health care: typology of barriers to health care access for low-income families. Ann Fam Med 2007;5:511-518.

70. Krieger N. Overcoming the absence of socioeconomic data in medical records: validation and application of a census-based methodology. Am J Public Health 1992;82:703-710. 
Supplemental online content for:

\section{Guideline-Concordant Treatment Among Elderly Women With HER2-Positive Metastatic Breast Cancer in the United States}

Ami M. Vyas, PhD, MS, MBA; Hilary Aroke, MD, PhD, MPH; and Stephen Kogut, PhD, MBA, RPh

J Natl Compr Canc Netw 2020;18(4):405-413

eTable 1: Treatment Algorithms Based on NCCN Guidelines for HER2-Positive Metastatic Breast Cancer 


\section{eTable 1. Treatment Algorithms Based on NCCN Guidelines for HER2-Positive Metastatic Breast Cancer}

HR-negative disease

(a) HER2-targeted therapy + chemotherapy

(i) Trastuzumab + taxane (docetaxel or paclitaxel)

OR

(ii) Pertuzumab + trastuzumab + taxane (docetaxel or paclitaxel)

OR

(iii) Trastuzumab + chemotherapya

OR

(iv) Other first-line therapies ([trastuzumab + paclitaxel \pm carboplatin] OR [trastuzumab + vinorelbine] OR [trastuzumab + capecitabine]) OR

HR-positive disease

(b) Trastuzumab only

(a) Aromatase inhibitors (anastrozole OR letrozole OR exemestane) OR

(b) Selective endocrine receptor modulators (tamoxifen OR toremifene)

OR

(c) Selective endocrine receptor downregulator (fulvestrant)

OR

(d) Other endocrine therapy (megestrol acetate OR fluoxymesterone OR ethinyl estradiol) OR

(e) Trastuzumab only OR

(f) HER2-targeted therapy + chemotherapy

(i) Trastuzumab + taxane (docetaxel or paclitaxel) OR

(ii) Pertuzumab + trastuzumab + taxane (docetaxel or paclitaxel) OR

(iii) Trastuzumab + chemotherapy

OR

(iv) Other therapies ([trastuzumab + paclitaxel + carboplatin] OR [trastuzumab + vinorelbine] OR [trastuzumab + capecitabine])

aThese included other chemotherapy agents for which claims were found: doxorubicin, gemcitabine, cyclophosphamide, fluorouracil. 